\title{
PERCEPÇÕES DE ENFERMEIROS DA ATENÇÃO PRIMÁRIA À SAÚDE SOBRE O CUIDADO A PACIENTES ONCOLÓGICOS
}

Anne Fayma Lopes Chaves

Uquiana Lucas Pereira ${ }^{2}$

Alexsandro Monteiro da Silva ${ }^{2}$

Luana Nunes Caldini ${ }^{3}$

Luana Cavalcante Lima ${ }^{4}$

Hérica Cristina Alves de Vasconcelos ${ }^{5}$ http://orcid.org/0000-0002-7331-1673 http://orcid.org/0000-0002-1253-1154 http://orcid.org/0000-0003-2556-047X http://orcid.org/0000-0001-9649-4081 http://orcid.org/0000-0001-8192-4974 http://orcid.org/0000-0001-9581-9147

Objetivo: Conhecer as percepções de enfermeiros sobre o cuidado a pacientes oncológicos, na Atenção Primária à Saúde. Método: Pesquisa descritiva, com abordagem qualitativa, realizada entre março e abril de 2019, em dez Unidades de Atenção Primária à Saúde, no município de Aracati, Ceará, Brasil. Os sujeitos da pesquisa foram 10 enfermeiros, sendo realizada entrevista semiestruturada registrada em formulário. Resultados: A partir das falas, emergiram três categorias: Percepções de enfermeiros sobre câncer e cuidados a pacientes oncológicos; Ações realizadas na atenção primária no cuidado a pacientes oncológicos; Dificuldades na assistência oncológica, na atenção primária. Conclusão: Os enfermeiros participantes visualizaram o câncer como doença estigmatizante, que interfere na qualidade de vida de pacientes e familiares, sendo importante a assistência oncológica no âmbito da atenção primária, principalmente, com auxílio de equipe multiprofissional.

Descritores: Atenção Primária à saúde; Oncologia; Cuidados de Enfermagem.

\section{PERCEPTIONS OF NURSES OF PRIMARY HEALTH CARE ABOUT THE CARE OF ONCOLOGICAL PATIENTS}

Objective: To know the perceptions of nurses about the care of cancer patients in Primary Health Care. Method: Descriptive research, with a qualitative approach, carried out between March and April 2019, in ten Primary Health Care Units, in the municipality of Aracati, Ceará, Brazil. The research subjects were 10 nurses, and a semi-structured interview was conducted, using a form. Results: From the speeches, three categories emerged: Nurses' perceptions about cancer and care for cancer patients: Actions carried out in primary care in the care of cancer patients; Difficulties in cancer care, in primary care. Conclusion: Participating nurses viewed cancer as a stigmatizing disease, which interferes with the life quality of patients and family members, and it is important to provide cancer care in primary care, especially with the help of a multidisciplinary team.

Descriptors: Primary health care; Oncology; Nursing Care.

\section{PERCEPCIONES DE ENFERMEROS DE ATENCIÓN PRIMARIA DE SALUD SOBRE LAATENCIÓN PARA PACIENTES ONCOLÓGICOS}

Objetivo: Conocer las percepciones de las enfermeras sobre la atención a pacientes con cáncer en Atención Primaria de Salud. Metodo: Investigación descriptiva, con enfoque cualitativo, realizada entre marzo y abril de 2019, en diez Unidades de Atención Primaria de Salud, en el municipio de Aracati, Ceará, Brasil. Los sujetos de investigación fueron 10 enfermeras, con una entrevista semiestructurada, utilizando una forma. Resultados: De los discursos, surgieron tres categorías: las percepciones de las enfermeras sobre el cáncer y la atención a los pacientes con cáncer; Acciones realizadas en atención primaria en la atención de pacientes con cáncer; Dificultades en la atención del cáncer, en atención primaria. Conclusión: Los enfermeros participantes vieron el cáncer como una enfermedad estigmatizante, que interfiere con la calidad de vida de los pacientes y sus familiares, y es importante brindar atención del cáncer en la atención primaria, especialmente con la ayuda de un equipo multidisciplinario. Descriptores: Atención primaria de salud; Oncología; Atención de enfermería.

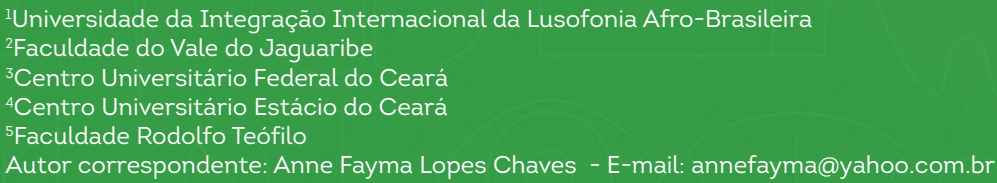




\section{INTRODUÇÃO}

O câncer é a segunda principal causa de morte no mundo, sendo responsável por uma estimativa de 9,6 milhões de mortes em 2018. Aproximadamente, $70 \%$ das mortes por câncer ocorrem em paises de baixa e média renda ${ }^{(1)}$. A estimativa brasileira para o biênio 2018-2019 era a ocorrência de 600 mil casos novos de câncer, com exceção do câncer de pele não melanoma, que se estimava em 420 mil casos novos de câncer ${ }^{(2)}$.

A neoplasia é uma doença que gera desafios para os pacientes e seus familiares. O paciente oncológico pode ter equilíbrio psicológico ameaçado pelo resultado do diagnóstico e as mudanças que serão necessárias, no decorrer da doença e do tratamento, incluindo alterações na autoestima. Diante disto, a adaptação ou o ajuste psicossocial ao câncer é um processo durante o qual cada pessoa procura controlar sofrimentos, resolver problemas específicos e alcançar algum controle sobre acontecimentos desencadeados pela doença ${ }^{(3)}$.

No que concerne ao tratamento do câncer, o paciente pode se defrontar com possiveis alterações na aparência física, limitações e impedimentos de atividades rotineiras que, muitas vezes, somam-se à quimioterapia, ao estigma da doença, à dificuldade para enfrentar o tratamento e seus efeitos colaterais, e à readaptação (3).

A qualidade de vida é fundamental para o ser humano, porém para a maioria dos pacientes com câncer essa qualidade (ou esse aspecto) vai gradativamente diminuindo, sendo importante que o doente receba assistência satisfatória quanto ao cuidado, tanto da família como dos profissionais que o assistem, visando suporte físico e emocional e para que possa vencer cada fase do tratamento (4).

A equipe de saúde que presta assistência ao paciente com câncer visa proporcionar o conforto deste, passando a aliviar as necessidades em relação aos aspectos psicológicos, sociais e espirituais ${ }^{(5)}$. Assim, o enfermeiro tem papel fundamental em todos os níveis de atenção à saúde, porém quando se trata da Atenção Primária à Saúde (APS), esse aspecto é mais complexo, haja vista que nesse nível de atenção as ações são desenvolvidas de modo individual e coletivas, bem como acompanhadas por equipes multiprofissionais que tem como objetivo principal a promoção da saúde, a prevenção de agravos, o diagnóstico, a reabilitação e a manutenção da saúde de pacientes (6).

Os enfermeiros desempenham diversos papéis na assistência oncológica no âmbito da APS, sendo um deles o de educador, o qual é essencial para a qualidade do cuidado. A atuação do enfermeiro no domicílio está muito voltada para ensinar o familiar os cuidados básicos a serem dis- pensados ao paciente ${ }^{(7)}$, ao longo do tratamento e processo de adoecimento

Estudo recente realizado com enfermeiros residentes de uma Escola de Enfermagem no Rio de Janeiro demonstra que a maioria destes (65\%) não conhece a Política Nacional de Atenção Oncológica (PNAO), evidenciando lacuna dessa categoria para a atenção oncológica, podendo indicar fragilidades no desenvolvimento de atividades na prática clínica ${ }^{(8)}$.

Outra pesquisa realizada com enfermeiras zambianas também revelou que a ausência de formação oncológica contribui para experiências negativas e impedem a prestação de cuidados de Enfermagem ideal, preocupação existente em países desenvolvidos e em desenvolvimento, o que evidencia a necessidade de capacitar esses profissionais para o atendimento a pacientes oncológicos ${ }^{(9)}$.

Diante desse contexto, surgiu a necessidade de realizar esta pesquisa, a qual teve como pergunta norteadora: Qual a percepção dos enfermeiros sobre o cuidado a pacientes oncológicos na APS? A relevância da pesquisa ora apresentada fundamenta-se no fato que ao conhecer a compreensão de enfermeiros da APS sobre o cuidado a pacientes oncológicos, poder-se-á contribuir para subsidiar esses profissionais e gestores da saúde no planejamento de ações centradas na assistência oncológica nesse nivel de atenção, visando capacitar os enfermeiros para que possam prestar assistência humana e qualificada. Logo, objetivou-se conhecer a percepção de enfermeiros da Atenção Primária à Saúde sobre o cuidado a pacientes oncológicos.

\section{MÉTODO}

\section{Tipo de estudo}

Pesquisa descritiva, com abordagem qualitativa.

\section{Participantes da pesquisa}

Os sujeitos da pesquisa foram enfermeiros que trabalhavam em 10 Unidades APS, três delas possuiam dois enfermeiros e as outras sete possuíam apenas um enfermeiro. Consideraram-se como critérios de inclusão: trabalhar na UAPS há pelo menos seis meses. Apenas um enfermeiro não contemplava esse quesito. Foram excluídos dois enfermeiros que estavam de licença (maternidade e saúde). Desse modo, totalizou-se 10 enfermeiros entrevistados.

\section{Local do estudo}

O estudo foi realizado em 10 Unidades de Atenção Primária a Saúde (UAPS), localizadas na zona urbana do município de Aracati - Ceará, Brasil. 


\section{Coleta dos dados}

A coleta das informações ocorreu entre março e abril de 2019. Inicialmente, foram realizadas visitas nas UAPS para averiguar as escalas dos enfermeiros e agendar a coleta. Os enfermeiros foram abordados antes ou após o serviço, de forma individual, em sala reservada, local em que foram apresentados os objetivos e benefícios da pesquisa e confirmada a participação mediante assinatura do Termo de Consentimento Livre e Esclarecido (TCLE).

A coleta se deu por meio de entrevista semiestruturada, sendo aplicado um formulário criado pelos próprios pesquisadores contendo duas partes: 1. Dados sociodemográficos e ocupacionais; e 2. Questões norteadoras da percepção sobre o cuidado a pacientes oncológicos na Atenção Primária à Saúde.

\section{Análises dos dados}

Para a análise das informações, utilizou-se o referencial de Bardin, nos quais foram submetidos a processos analíticos preconizados pela Análise de Conteúdo Temático em quatro etapas: Pré-análise, exploração do material, tratamento dos resultados obtidos e interpretação ${ }^{(10)}$. Logo, emergiram três categorias temáticas: 1. Percepções de enfermeiros sobre o câncer e os cuidados a pacientes oncológicos; 2. Ações desenvolvidas durante o cuidado a pacientes oncológicos na APS; 3. Dificuldades da assistência oncológica na APS.

\section{Procedimentos éticos}

A pesquisa obedeceu à Resolução no 466/12, do Consetho Nacional de Saúde que envolve a pesquisa com seres humanos, sendo aprovado pelo Comitê de Ética em Pesquisa, do Instituto do Câncer do Ceará (ICC), conforme parecer № 3.178.683. Ressalta-se que o projeto foi implementado após anuência formal da Secretaria da Saúde do município.

A pesquisa manteve o anonimato com relação aos nomes dos entrevistados, os quais foram codificados com nomes de flores.

\section{RESULTADOS}

Esse estudo teve 10 participantes enfermeiros, sendo dois homens e oito mulheres, com idades entre 24 e 43 anos. Dentre estes, sete concluíram curso de especialização nas seguintes áreas: Enfermagem Obstétrica (três), Saúde da Família (três) e Estomatoterapia (um).

$\mathrm{Na}$ fase de interpretação, os dados referentes a assistência oncológica na atenção primária à saúde, sob a perspectiva dos profissionais de enfermagem atuantes nas APS, foram agrupados em categorias. As mesmas serão apresentadas a seguir.

Percepções de enfermeiros sobre o câncer e os cuidados a

\section{pacientes oncológicos}

Foi unânime entre os relatos dos enfermeiros que o câncer ainda é uma doença bastante estigmatizada, sendo vista como doença fatal, que desencoraja qualquer expectativa de melhora ou cura, afetando não apenas o paciente, mas a família deste, atingindo aspectos biopsicossociais:

Em minha percepção, vejo o câncer como o fim de uma vida, um ciclo que se fecha, uma doença fatal (Flor do Sertão).

O câncer é uma doença devastadora, ele não afeta só o paciente, mas sim a todos (familia, amigos). A cada dia, aumentam os casos de câncer em todo o mundo, e as pessoas, mesmo diante disso, deixam para se cuidar em último caso, levando, assim, muitas vezes, a um diagnóstico tardio e sem muitas chances de tratamento, em alguns casos (Amélia).

Quando interrogados sobre o cuidado oncológico nas APS, a maioria dos enfermeiros expôs ser de grande relevância a assistência a essa clientela específica ,-sobretudo por uma equipe multiprofissional, que seja capaz de atender às necessidades básicas, visto que eles necessitam de atenção especial, em virtude das mais diversas intercorrências que irão surgir em decorrência do estadiamento natural da doença.

Eu acredito que a familia em si, e não só o paciente, precisa de cuidados na unidade básica, para se ter melhor qualidade de vida para o paciente-familia, é onde entra o apoio da equipe multiprofissional (Lírio).

Eu penso que é na rede primária que podemos detectar qualquer sinal de agravamento da doença e suas complicações, para sinalizarmos ao paciente que algo não vai bem e, juntos traçarmos uma linha de atendimento para o tratamento (Camélia).

\section{Ações desenvolvidas durante o cuidado a pacientes onco- lógicos na APS}

Nesta categoria, observou-se que as ações mais citadas pelos entrevistados diante da assistência oncológica estiveram relacionadas a manter hábitos de vida saudáveis e trabalhar o estado psicológico, haja vista a fragilidade emocional desses pacientes frente à doença. 
Eu oriento de acordo com a realidade de cada um, e tentamos resgatar, primeiramente, a autoconfiança e a autoestima, para que se dê a continuidade ao tratamento; em seguida, orientamos sobre os benefícios de aderir a hábitos de vida saudável, desde a alimentação, da prática de atividade física até a aderência do grupo (Papoula).

Nós da equipe orientamos a esses pacientes para não fumar, não ingerir bebidas alcóolicas, dietas sem gorduras, frituras, fazer atividade física e seguir as orientações dos profissionais de saúde (Camélia).

Eu, enquanto enfermeira da UBS realizo ações voltadas, primeiramente, para questões emocionais do paciente, buscando resgatar a autoconfiança e autoestima deles. para que, posteriormente, o paciente prossiga com a continuidade do tratamento, pois eu percebo que eles ficam muito fragilizados (Papoula).

Apesar de os enfermeiros reconhecerem a importância da assistência oncológica na APS, relataram a não realização de ações a esse público, bem como o desconhecimento de programas e protocolos do Ministério da Saúde do Brasil voltados para assistência a pacientes oncológicos.

Nunca realizei ações voltadas para assistência ao paciente oncológico. Costumo realizar palestras, rodas de conversas para incentivar a prevenção, não especificamente aos pacientes com câncer (Lótus).

Eu não costumo realizar ações para esse público com câncer, somente realizo acompanhamento ambulatorial (consultas, orientações e análises de exames) (Girassol).

\section{Dificuldades da assistência oncológica na APS}

Percebeu-se que as maiores dificuldades enfrentadas pelos enfermeiros perante a assistência à clientela com câncer na APS, foi a limitação dos exames disponibilizados e dos recursos acessíveis na unidade para a melhoria da assistência; além da necessidade de educação permanente sobre a temática, pois os participantes não se consideravam capacitados para o atendimento adequado.
A minha grande dificuldade é quando é solicitado ao paciente exames mais complexos (ressonância, tomografia, densitometria óssea), acaba que a demora a ser chamado e, muitas vezes, não chega nem a fazer, por falta de recursos próprios também. Penso que a maior dificuldade seja a marcação e a lentidão de exames mais complexos (Crisântemo).

Apesar de me achar preparada, eu encontro dificuldades em desenvolver um bom trabalho, em virtude das condições oferecidas na unidade básica (Papoula).

Eu aprendi muito pouco durante a graduação sobre os cuidados aos pacientes com câncer, raramente foram abordadas questões sobre esse processo de cuidar e nem foram favorecidas vivências que nos aproximasse dessas realidades (Lírio).

\section{DISCUSSÃO}

As percepções dos enfermeiros apontaram o câncer como situação de mutilação e abreviamento da vida. A doença, ainda, está associada à severa condição fatal, no entanto, quando se interroga enfermeiros, vislumbra-se a coerência em cuidar e não apenas buscar a cura ${ }^{(8)}$. Esse pensamento vai ao encontro da ideologia dos cuidados paliativos, a qual propõe oferecer conforto e alívio necessário para minimizar o sofrimento do paciente, visando melhor qualidade de vida até os últimos dias ${ }^{(5)}$.

Nessa perspectiva, o aprofundamento e a obtenção de competências na área de cuidados paliativos são demandas legítimas, considerando grande quantidade de pessoas portadoras de câncer, as quais requerem cuidados centrados no controle de sintomas, na assistência psicossocial e espiritual, e na atenção devida aos familiares (11)

A fim de desenvolver assistência qualificada, a maioria dos enfermeiros relatou a importância da atuação de equipe multiprofissional no cuidado aos pacientes oncológicos. $\mathrm{O}$ impacto da equipe multidisciplinar no tratamento do câncer é reconhecido, sendo capaz de atuar na prevenção, no controle, na avaliação diagnóstica, no tratamento, na reabilitação e no atendimento aos familiares ${ }^{(12)}$.

A atuação da equipe multidisciplinar, no âmbito da APS, remete, também, ao cuidado paliativo no domicílio, o qual visa minimizar a fragilidade do sofrimento com a doença e assegurar a dignidade no processo de morrer (13). Pesquisa que investigou a percepção de pacientes com câncer apontou 
vantagens do cuidado oncológico no domicílio, como liberdade, conforto, autonomia e fortalecimento do vínculo com a equipe de saúde ${ }^{(14)}$

Constatou-se que as ações mais realizadas pelos enfermeiros da APS consistiam na manutenção de hábitos de vida saudável e no apoio psicológico. A promoção do desenvolvimento de hábitos saudáveis favorece não apenas a prevenção, como pode ser capaz de minimizar os grandes danos causados pelo tratamento terapêutico, principalmente os efeitos colaterais ou de toxicidade, ocasionados pela quimioterapia ${ }^{(15)}$

Outras evidências destacam, ainda, que o fator psicológico é bastante afetado em virtude das inúmeras mudanças e adaptações necessárias durante o processo terapêutico, as quais afetam diretamente a autoestima do paciente ${ }^{(3)}$.

O enfermeiro que presta cuidado a pacientes oncológicos não deve se limitar apenas aos cuidados gerais, precisa ter competência para atuar diante das reações esperadas, decorrentes do tratamento. Estudo que envolveu 15 enfermeiros da Estratégia Saúde da Família (ESF) de três municípios do norte do Estado do Rio Grande do Sul, Brasil, evidenciou lacunas no conhecimento destes, diante do atendimento a pacientes portadores de neoplasias, comprometendo a assistência prestada e gerando risco de complicações advindas do tratamento ${ }^{(16)}$

Diversas são as consequências do diagnóstico e do tratamento para o paciente oncológico, sendo imprescindivel que a equipe de saúde, em especial o enfermeiro, atue ativamente na identificação precoce de complicações físicas e psicológicas, possibilitando previamente o estabelecimento de um plano de cuidado individualizado centrado na melhoria da qualidade de vida das pessoas com câncer ${ }^{(17)}$

Dado preocupante encontrado nesta pesquisa foi o relato de enfermeiros sobre a não realização de ações específicas com esses pacientes, bem como o desconhecimento de programas e políticas para pacientes oncológicos. Recentemente, o Ministério da Saúde implantou a Política Nacional para Prevenção e Controle do Câncer, a qual estabelece diretrizes nacionais para a prevenção e o controle do câncer, estimulando a atenção integral e articulando as diversas ações, nos três níveis de gestão do Sistema Único de Saúde (SUS). O objetivo central da política é contribuir para melhoria da qualidade de vida dos usuários com câncer, por meio de ações de promoção da saúde, prevenção de riscos e agravos, detecção precoce, tratamento oportuno e cuidados paliativos ${ }^{(18)}$.

Pesquisa recente apresentou discussão quanto à inserção do conhecimento das políticas públicas em oncologia, na formação de estudantes de Enfermagem, motivando reflexões de como esse conteúdo na academia poderia con- tribuir para melhorar as práticas na assistência a pacientes oncológicos ${ }^{(19)}$

Nessa perspectiva, é urgente que enfermeiros busquem a reformulação do pensamento crítico e percebam a necessidade de conhecimentos que os capacitem à assistência a pacientes oncológicos, provendo, assim, meios e recursos que favoreçam esse cuidado em todos os níveis de atenção à saúde.

A pesquisa em tela constatou o despreparo e a carência de conhecimentos dos enfermeiros sobre a oncologia. Pesquisa qualitativa que envolveu 18 enfermeiros atuantes em unidades de internação e ambulatórios oncológicos no Estado do Rio Grande do Sul evidenciou déficit de conhecimento sobre a temática, desde a formação acadêmica até a atuação dos profissionais no mercado de trabalho, mostrando que a precariedade da educação permanente pode repercutir em uma prática competente ${ }^{(20)}$

Diante desse contexto, reconhece-se a necessidade da inserção do conteúdo sobre assistência de Enfermagem em oncologia nos currículos de graduação em Enfermagem e pós-graduação lato sensu (especialização). Portanto, para que isso aconteça, é preciso que esteja contextualizada no Projeto Pedagógico do Curso (PPC), garantindo, assim, formação com benefício para prática, difundindo o conhecimento entre estudantes de graduação, para que possam melhor lidar com o câncer, nas esferas técnico-científica e emocional ${ }^{(20)}$, nos diversos campos de atuação que forem seguir após o término da graduação.

A ausência de educação permanente dos enfermeiros nas unidades de APS, também tem sido um dos fatores que contribui para o despreparo em atendimento qualificado a pacientes oncológicos e suas famílias. Estudo realizado em Diadema, São Paulo, com 70 enfermeiros, revelou que a educação permanente é um dos fatores primordiais para o controle do câncer, bem como a qualificação profissional e especialização, visto que tais conhecimentos são capazes de estabelecerem a detecção do câncer, a orientação oportuna a pacientes e o encaminhamento para unidade adequada, quando se fizer necessário (21)

Somado a esse déficit de conhecimento que permeia os enfermeiros da APS, no que diz respeito à assistência aos pacientes com câncer, foi citado pelos enfermeiros entrevistados nesta pesquisa que as principais dificuldades encontradas na assistência oncológica na APS consistia em fatores organizacionais, que vão de encontro ao que é preconizado pelos principios do SUS.

Dentre os desafios apontados como empecilhos para eficiência de estratégias no diagnóstico e tratamento do câncer, tem-se a escassez de recursos na UAPS e a limitação dos 
exames disponibilizados para os pacientes, configurando fragilidade da rede de atenção a pacientes oncológicos.

Essa vulnerabilidade da rede de atenção à saúde e a carência de recursos financeiros também foram reconhecidos pelos profissionais da saúde, dentre eles enfermeiros, e usuários como fatores que influenciam negativamente o acesso aos serviços de saúde. A limitação de recursos e os problemas organizativos da Estratégia Saúde a Família (ESF) refletem diretamente na assistência prestada, desde o acesso (marcação de consultas, acolhimento) até a integralidade do cuidado (fluxo de atendimento: referência e contrarreferência), dificultando o atendimento das necessidades da população, podendo gerar risco à saúde e diminuindo a satisfação em relação aos serviços prestados ${ }^{(22)}$.

Os processos organizativos do SUS, ainda, tateiam entre a descentralização que fragmenta os serviços e a regionalização que deve uni-los em rede integrada. No entanto, o processo de regionalização não está efetivamente estabelecido, sendo imprescindivel intensificar a relação entre a União, estados e municípios, no que diz respeito aos compromissos e às responsabilidades, haja vista que esse processo envolve dinâmicas socioeconômicas e políticas ${ }^{(23)}$

No contexto da atenção oncológica, além desses problemas que repercutem indiretamente na assistência dos pacientes, estes ainda enfrentam as dificuldades vivenciadas ao longo dos itinerários para o diagnóstico e terapêutica, como sentimentos negativos diante da doença, ausência de acompanhamento de familiares durante o tratamento e insuficiência de recursos financeiros para custeá-la ${ }^{(24)}$. Esses fatores também precisam ser ponderados pelos enfermeiros assistenciais e gestores das unidades de saúde, a fim de minimizar o impacto da doença durante essa jornada.

\section{Limitação do estudo}

Aponta-se como limitação da pesquisa o fato de o estudo ter sido realizado apenas em um município do Ceará, fator que impede generalizações dos resultados.

\section{Contribuições para a prática}

Os resultados apresentados neste estudo indicaram que, embora a assistência oncológica seja importante para os enfermeiros atuantes na atenção primária, os próprios participantes mencionaram despreparo e carência de conhecimentos sobre oncologia. Destarte, percebe-se a necessidade de aprimoramento da qualificação desses enfermeiros. Assim, reiterando a relevância dita outrora, os resultados poderão subsidiar gestores no planejamento e ações centradas na assistência oncológica que visem capacitar os enfermeiros para a prestação de assistência segura, humana e qualificada.

\section{CONCLUSÃO}

Os enfermeiros participantes visualizaram o câncer como uma doença estigmatizante que interfere na qualidade de vida de pacientes e familiares, sendo relatado por eles a importância da assistência oncológica, no âmbito da APS, principalmente, com o auxílio de uma equipe multiprofissional.

As ações mais realizadas pelos enfermeiros deste estudo, consistiam em promover hábitos de vida saudáveis e apoio psicológico. Apesar disso, visualizou-se a lacuna de conhecimento dos enfermeiros envolvidos, quanto a protocolos e programas que auxiliam essa assistência, bem como as barreiras vivenciadas na prática clínica, como limitação dos exames disponibilizados e necessidade de educação permanente.

Dessa forma, os enfermeiros que atuam na APS necessitam de aprimoramento na assistência ao cliente oncológico e sua família, sendo necessário que esse processo inicie desde a graduação, com a inserção do conteúdo da assistência de enfermagem em oncologia nos currículos atuais.

\section{CONTRIBUIÇÃO DOS AUTORES:}

Pereira UL e Chaves AFL participaram da concepção, análise, interpretação dos dados, redação do artigo e revisão crítica e final. Da Silva AM, Lima LC, Caldini LN e De Vasconcelos HCA contribuíram com a revisão crítica e final.

\section{REFERÊNCIAS}

1.World Health Organization (WHO). Cancer [Internet]. [cited 2020 Feb 19]. Available from: http://www.who.int/mediacentre/factsheets/ fs297/en/

2.Santos MO. Estimate 2018: Cancer Incidence in Brazil. Revista Brasileira de Cancerologia [Internet]. 2018 [cited 2020 Feb 19];64(1):119-120 Available from: http://wwwl.inca.gov.br/rbc/n_64/v01/pdf/15-resenha-estimativa-2018-incidencia-de-cancer-no-brasil.pdf

3.Leite MAC, Nogueira DA,Terra FS. Avaliação da autoestima em pacientes oncológicos submetidos a tratamento quimioterápicol. Rev. Latino-Am. Enfermagem [Internet]. 2015 [cited 2018 Set 10];23(6):82-89. Available from: http://www.scielo.br/pdf/rlae/v23n6/pt_0104-1169-rlae-23-06-01082.pdf 
4.Acioli S, Kebian LVA, Faria MGA, Ferraccioli P, Correa VAF. Práticas de cuidado: o papel do enfermeiro na atenção básica. Rev enferm UERJ [Internet]. 2014 [cited 2018 Out 10];22(5):37-42. Available from: http://www.facenf.uerj.br/v22n5/v22n5a09.pdf

5.Fernandes MA, Evangelista CB, Platel ICS, Angra G, Lopes MS, Rodrigues FA. Percepção dos enfermeiros sobre o significado dos cuidados paliativos em pacientes com câncer terminal. Ciênc. saúde coletiva [Internet]. 2013 [cited 2018 Out 15];18(9):89-96. Available from: http://www.scielo.br/scielo.php?script=sci_arttext\&pid=S1413-81232013000900013

6.Soares FS, Périco LAD, Dias VRF. A complexidade do trabalho do enfermeiro na Atenção Primária à Saúde. Rev. Bras. Enferm. [Internet]. 2018 [cited 2019 Jul 21];71(1):52-57. Available from: http://www.scielo.br/pdf/reben/v7lsl/pt_0034-7167-reben-71-sl-0704.pdf

7.Vale JMM, Marques Neto AC, Santos LMS, Santana ME. Educação em saúde ao familiar cuidador de adoecidos em cuidados paliativos oncológicos domiciliares. Enferm. Foco [Internet]. 2019 [cited 2020 Feb 19];10(2):52-57. Available from: http://revista.cofen.gov.br/index. php/enfermagem/article/view/1684/519

8.Lins F, Souza SR. Formação dos Enfermeiros para o cuidado em oncologia. Rev Enferm. UFPE [Internet]. 2018 [cited 2018 Set 14]:12(1):6674. Available from: https://periodicos.ufpe.br/revistas/revistaenfermagem/article/viewFile/22652/25858

9.Maree JE, Mulonda JK. Caring for Patients with Advanced Breast Cancer: The Experiences of Zambian Nurses. Asia Pac J Oncol Nurs [Internet]. 2017 [cited 2020 Feb 19];4(1):23-28. Available from: https://www.researchgate.net/publication/313126999_Caring_for_Patients_with_Advanced_Breast_Cancer_The_Experiences_of_Zambian_Nurses

10.Bardin L. Análise de conteúdo. Tradução Luis Antero Reto e Augusto Pinheiro. São Paulo: Edições 70, 2011. Available from: https://www. scielo.br/scielo.php?script=sci_nlinks\&ref=000179\&pid=S00347612201300040000400003\&lng=pt

11.Caldas GHO, Moreira SNT, VilarMJ. Cuidados paliativos: Uma proposta para o ensino da graduação em Medicina. Rev. Bras. Geriatr. Gerontol [Internet]. 2018 [cited 2010 Dez 20];21(3):69-80. Available from: http://www.scielo.br/scielo.php?pid=S1809=98232018000300261-Escriptsci_arttextEtlng=pt

12.Silva RCV, Sant'Ana RSE, Cardoso MBR, Alcântara LFFL. Cancer care and interdisciplinary practice. Cad. Saúde Pública [Internet]. 2019 [cited 2019 Fev 20];35(1):1-3. Available from: http://www.scielo.br/scielo.php?pid=S0102-311X2019000108001Escript=sci_arttext\&tlng=en 13.Oliveira MBP, Souza NR, Bushatsky M, Dâmaso BFR, Bezerra DM, Brito JA. Oncological homecare: family and caregiver perception of palliative care. Esc Anna Nery [Internet]. 2017 [cited 2019 Fev 01];21(2):1-6. Available from: http://www.scielo.br/scielo.php?pid=S1414-814520170002002028script=sci_abstract

14.Matos MR, Muniz RM, Viegas AC, Przylynski DS, Holz AW. Significado da atenção domiciliar e o momento vivido pelo paciente oncológico em cuidados paliativos. Rev. Eletr. Enf. [Internet]. 2016 [ cited 2019 Dez 20]:18(1):10. Available from: https://revistas.ufg.br/fen/article/ view/35061/22001

15.Varejão CS, Borges GG, Nunes LMP, Silvino ZR, Espírito Santo FH, Christovam BP. Terapêutica Oncológica para Enfermeiros e Farmacêuticos. Rev enferm UFPE on line [Internet]. 2014 [cited 2018 Mar 10];8(12):6-7. Available from: https://periodicos.ufpe.br/revistas/revistaenfermagem/article/download/10193/1075

16.Cruz FS, Rossato RG. Care Given to Cancer Patients Undergoing Chemotherapy: Knowledge of Family Health Strategy Nurses. Revista Brasileira de Cancerologia [Internet]. 2015 [cited 2019 Dez 21];61(4): 35-41. Available from: http://wwwl.inca.gov.br/rbc/n_61/v04/ pdf/04-artigo-cuidados-com-o-paciente-oncologico-em-tratamento-quimioterapico-o-conhecimento-dos-enfermeiros-da-estrategia-saude-da-familia.pdf

17.Simão DAS, Aguiar ANA, Souza RS, Captein KM, Manzo BF, Teixeira AL. Qualidade de vida, sintomas depressivos e de ansiedade no início do tratamento quimioterápico no câncer: desafios para o cuidado. Enferm. Foco [Internet]. 2017 [cited 2020 Feb 20];8 (2): 82-86. Available from: http://revista.cofen.gov.br/index.php/enfermagem/article/view/874/385

18.Mendes EC, Vasconcellos LCF. Cuidados paliativos no câncer e os principios doutrinários do SUS. Saúde Debate [Internet]. 2015 [cited 2019 Fev 20];39(106):81-92. Available from: http://www.scielo.br/pdf/sdeb/v39n106/0103-1104-sdeb-39-106-00881.pdf

19.Oliveira AM, Silva KSEM. Formação do enfermeiro: políticas públicas na atenção oncológica. Enferm. Foco [Internet]. 2018 [cited 2019 Dez 21];9(3):48-52 Available from: http://revista.cofen.gov.br/index.php/enfermagem/article/view/1175/460

20.Luz KR, Vargas MAO, Rosa LM, Schmitt PH. Enfermeiros na atenção oncológica: conhecimento na prática do cuidado. Rev enferm UFPE on line [Internet]. 2016 [cited 2019 Jul 23]:10(9):69-76. Available from: https://periodicos.ufpe.br/revistas/revistaenfermagem/article/ download/11418/13204

21.Teixeira MS, Goldman RE, Gonçalves VCS, Gutiérrez MGR, Figueiredo EM. Atuação do enfermeiro da Atenção Primária no controle do câncer de mama. Acta Paul Enferm [Internet]. 2017 [cited 2018 ago 29];30(1):1-7. Available from: http://www.scielo.br/pdf/ape/v30nl/19820194-ape-30-01-0001.pdf

22.Viegas APB, Carmo RF, Da Luz ZMP. Factors associated to the access to health services from the point of view of professionals and users of basic reference unit. Saúde Soc. São Paulo [Internet]. 2015 [cited 2019 Dez 15];24(1):01-12. Available from: http://www.scielo.br/pdf/sausoc/v24nl/0104-1290-sausoc-24-1-0100.pdf

23.Carvalho ALB, Jesus WLA, Senra IMVB. Regionalization in the SUS: implementation process, challenges and perspectives in the critical view of system managers. Ciência \& Saúde Coletiva [Internet]. 2017 [cited 2019 Dez 15];22(4):55-64. Available from: http://www.scielo.br/ $\mathrm{pdf} / \mathrm{csc} / \mathrm{v} 22 \mathrm{n} 4 / 1413-8123-\mathrm{csc}-22-04-1155 . \mathrm{pdf}$

24.Teston EF, Fukumori EFC, Benedetti GMS, Spigolon DN, Costa MAR, Marcon SS. Feelings and difficulties experienced by cancer patients along the diagnostic and therapeutic itineraries. Esc Anna Nery [Internet]. 2018 [cited 2019 Jan 14];22(4): 1-8. Available from: http://www. scielo.br/pdf/ean/v22n4/1414-8145-ean-22-04-e20180017.pdf 